\title{
Hypopigmented Scar following Transradial Catheterization: An Extremely Rare Local Site Complication
}

\author{
Santosh Kumar Sinha, Puneet Aggarwal, Mukesh Jitendra Jha, Vikas Mishra \\ Department of Cardiology, LPS Institute of Cardiology, Ganesh Shankar Vidyarthi Memorial Medical College, Kanpur, \\ Uttar Pradesh, India.
}

\section{Corresponding Author:}

Dr. Santosh Kumar Sinha

Email: fionasan@rediffmail.com

This is an Open Access article distributed under the terms of the Creative Commons Attribution License (creativecommons.org) licenses/by/3.0).

Received

Accepted

Published

April 29, 2020

September 3, 2020

December 5, 2020

\begin{abstract}
Background: Transradial route is predominant access site for cardiac catheterization procedures but there is little data about hypopigmented scar at puncture site post procedure. Case Report: A 64-year-old male presented with inferior wall ST segment elevation myocardial infarction. Primary percutaneous coronary intervention was performed of left circumflex artery followed by transradial band application at puncture site for 120 minutes. At 8-week follow-up, a hypopigmented scar was noted over puncture site which is an exceedingly rare complication at local site. Conclusion: Prolonged compression at higher pressure by TR band resulted into pressure necrosis leading into hypopigmented scar. Adopting a "patent hemostasis" protocol could have averted such complication.

Keywords: Cardiac Catheterization, Hemostasis, Percutaneous Coronary Intervention, ST Elevation Myocardial Infarction, Punctures.
\end{abstract}

\section{Case Description}

Transradial route is increasingly being adopted for both diagnostic and therapeutic purposes ever since first reported in 1989 because of its natural advantages like lower vascular complication, faster ambulation, patient preference, and lower morbidity and mortality. Although rare albeit complications like spasm, haematoma, perforation, dissection, and persistent radial artery occlusion have been reported. Another extremely rare reported complication is hypopigmented scar.

A 64-year-old male presented with diaphoresis and retrosternal chest pain of 3 hour duration. On clinical examination, his vitals were stable. Electrocardiogram revealed ST elevation in II, III and aVF with reciprocal changes in lead I and aVL. Therefore, he was diagnosed as inferior wall ST segment elevation myocardial infarction. Smoking, dyslipidemia and diabetes were his risk factors. Echocardiogram revealed regional wall motion abnormality involving left circumflex (LCx) territory with mild systolic dysfunction. He was preloaded with $90 \mathrm{mg}$ ticagrelor, 40 $\mathrm{mg}$ rosuvastatin, and $325 \mathrm{mg}$ chewable aspirin. Primary percutaneous coronary intervention was planned through transradial route. After puncturing right radial artery using a Teflonsheathed needle, $6 \mathrm{~F}$ introducer sheath (Terumo Inc, Japan) was introduced. Coronary angiogram was performed using Tiger catheter (Terumo Inc, Japan) after administering cocktail containing 200 $\mu \mathrm{g}$ nitroglycerine, $2.5 \mathrm{mg}$ diltiazem, and $2500 \mathrm{IU}$ unfractioned heparin which revealed thrombotic lesion in dominant proximal LCx causing subtotal occlusion with Thrombosis In Myocardial Infarction (TIMI) I flow. 2500 IU unfractioned heparin was further administered. Lesion in $\mathrm{LCx}$ was successfully stented using $3 \times 23 \mathrm{~mm}$ Endeavour Resolute Zotarolimus eluting stent (Medtronic Inc; USA) at $14 \mathrm{~atm}$ pressure achieving TIMI III flow. After placing transradial band (TR band, Terumo; 


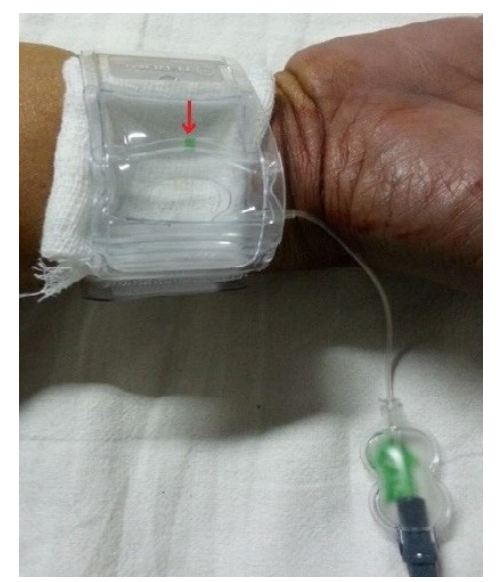

Fig.1: Inflated TR band with small green box indicator present on it.

Japan) at the puncture site and inflating it partially, radial sheath was removed. It was decompressed to allow some back bleed and further $20 \mathrm{ml}$ of air was introduced using $10 \mathrm{ml}$ syringe in inflatable cuff of TR band which was kept for 120 minutes to achieve proper hemostasis [Fig.1]. Once proper hemostasis was secured, TR band was replaced by light pressure bandage which was removed next day and patient was discharged in stable condition with appropriate advice. At 8-week follow-up, a hypopigmented scar was noted over puncture site [Fig.2]. Prolonged compression at higher pressure by TR band resulted into pressure necrosis leading into hypopigmented scar. Adopting a "patent haemostasis" protocol could have averted such complication [1].

Hypopigmented scar, an exceedingly rare complication (4 cases among 20,000 procedures),

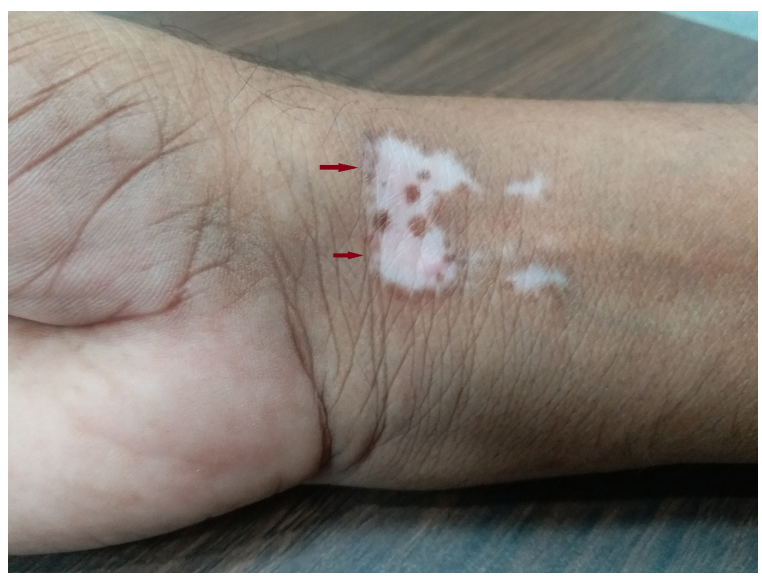

Fig.2: Hypopigmented scar seen over the radial puncture site on follow-up at 8-weeks.

may result from undue compression because of prolonged inflation at higher pressure by TR band. TR band should be placed little proximal to insertion site of sheath and inflated with $13-15 \mathrm{ml}$ of air while radial artery should be checked at least once every 20 minutes by palpation and colour of palm to ensure its patency.

Contributors: SKS: Manuscript writing, and literature review; PA: manuscript editing, and references. MJJ, VM: critical inputs into the manuscript. SKS will act as a study guarantor. All authors approved the final version of this manuscript and are responsible for all aspects of the study.

Funding: None; Competing interests: None stated.

\section{References}

1. Pancholy SB, Bernat I, Bertrand OF, Patel TM. Prevention of radial artery occlusion after transradial catheterization: The PROPHET-II Randomized Trial. JACC Cardiovasc Interven. 2016;9:1992-1999. 